\title{
Pharmacokinetic Limitations on Effects of an Alpha7-Nicotinic Receptor Agonist in Schizophrenia: Randomized Trial with an Extended-Release Formulation
}

\author{
William R Kem', Ann Olincy², Lynn Johnson², Josette Harris², Brandie D Wagner ${ }^{3}$, Robert W Buchanan ${ }^{4}$, \\ Uwe Christians $^{5}$ and Robert Freedman*,2 \\ 'Department of Pharmacology and Therapeutics, University of Florida College of Medicine, Gainesville, FL, USA; ${ }^{2}$ Department of Psychiatry F-546, \\ University of Colorado School of Medicine, Aurora, CO, USA; ${ }^{3}$ Department of Biostatistics and Informatics, Colorado School of Public Health, Aurora, \\ CO, USA; ${ }^{4}$ Maryland Psychiatric Research Center, Department of Psychiatry, University of Maryland School of Medicine, Baltimore, MD, USA; \\ ${ }^{5}$ Department of Anesthesiology, University of Colorado School of Medicine, Aurora, CO, USA
}

\begin{abstract}
The aim of the trial was to assess whether extending plasma levels of the alpha7-nicotinic acetylcholine receptor (nAChR) agonist 3-(2,4dimethoxybenzylidene)-anabaseine (DMXB-A) over time enhances its cognitive effects in schizophrenia. Both smoking and non-smoking patients were studied, to determine whether effects differ between these two groups. Forty-three smokers and thirty-seven non-smokers who met DSM-IV criteria for schizophrenia were enrolled in a double-blind, randomized, placebo-controlled I month trial. DMXB-A $150 \mathrm{mg}$ was formulated with hypromellose to produce extended release over $4 \mathrm{~h}$ and administered four times daily. The primary outcome (the Neurocognitive Composite of the MATRICS Consensus Cognitive Battery) and secondary outcomes (the MATRICS Attention-Vigilance Domain and P50 gating), showed no significant effect. Plasma levels were obtained $2.5 \mathrm{~h}$ post administration. In non-smokers, levels were similar to those reached transiently with 75-150 mg DMXB-A immediate-release formulations twice daily, which were earlier shown to be effective doses. However, the extended-release formulation produced no cognitive or clinical effect either in non-smokers or smokers. The I0-fold lower DMXB-A plasma levels in smokers suggest that chronic smoking enhances DMXB-A metabolism. Pro-cognitive effects of DMXB-A may result from transient increases in cell signaling that are limited by receptor tachyphylaxis. Future efforts to improve cognition in schizophrenia by enhancing alpha7 nAChR function may require consideration of these pharmacokinetic limitations.

Neuropsychopharmacology (20I8) 43, 583-589; doi:I0.1038/npp.20 I7.I82; published online 20 September 2017
\end{abstract}

\section{INTRODUCTION}

The alpha7-nicotinic acetylcholine receptor (nAChR) has received increasing interest as a target for the development of new drugs that will ameliorate cognitive deficits in people with schizophrenia. Several companies developed drug candidates, some with successful initial trials (Lieberman et al, 2013; Keefe et al, 2015; Haig et al, 2016a). However, none of these candidates has shown significant cognitive effects in schizophrenia in larger trials (Umbricht et al, 2014; Walling et al, 2016; Haig et al, 2016b). We earlier showed cognitive effects with single-dose administration of the partial agonist 3-(2,4-dimethoxybenzylidene)-anabaseine (DMXB-A) using the Repeatable Battery for the Assessment of Neurocognitive Status, and after 1 month administration in the Attention and Executive Function Domains of the

\footnotetext{
*Correspondence: Dr R Freedman, Department of Psychiatry F-546, University of Colorado School of Medicine Anschutz Medical Campus, Box C-268-7I, Aurora, CO 80045, USA, Tel: +720 224 4638, Fax: +303 724 4956, E-mail: Robert.Freedman46@gmail.com

Received 2 May 2017; revised 10 August 2017; accepted 14 August 2017; accepted article preview online 21 August 2017
}

MATRICS Consensus Cognitive Battery (Olincy et al, 2006; Freedman et al, 2008). Unlike the relatively long plasma halflife drug candidates in industry development that were designed to permit daily dosing, DMXB-A has an exceptionally short half-life, $\sim 60 \mathrm{~min}$ (Kitigawa et al, 2003). A short half-life would seem to be an undesirable property for a drug developed to treat a chronic illness. However, the alpha7$\mathrm{nAChR}$ displays very rapid desensitization with even brief exposure to agonists (Peng et al, 1994; Lopez-Hernandez et al, 2009). Therefore, a short half-life drug that can produce a large transient stimulation of the receptor might actually produce a greater pro-cognitive effect relative to a drug producing a more constant elevation in receptor stimulation.

Differences in effect between drugs depend on both their pharmacodynamic and pharmacokinetic (PK) properties. Alpha7-nicotinic agonists differ not only in their half-lives but also in their relative effectiveness as agonists and antagonists, as all nAChR agonists, including acetylcholine itself, have both properties (Briggs et al, 1998; Papke et al, 2009). Therefore, comparison between drugs with different pharmacodynamic properties cannot answer the question of which PK properties are most desirable. This question has 
importance for developing drugs with optimal effectiveness. To determine which PK properties are optimal for an alpha7 agonist, we have tested an extended-release formulation of DMXB-A, the same partial agonist that we initially had tested as an immediate-release formulation.

DMXB-A was previously tested in a methylcellulose-filled gelatin capsule formulation that allows its immediate release in the stomach and maximal absorption in about $60 \mathrm{~min}$ (Kitagawa et al, 2003). To extend patient exposure to the compound, we reformulated it in hypromellose-filled capsules. Hypomellose swells into a matrix in the gastric fluid. DMXB-A is then released more slowly as the matrix is physically degraded during digestion. The process occurs more quickly in the alkaline duodenum than in the stomach, and the drug is released over $4 \mathrm{~h}$ in most patients. This extended-release formulation enables us to determine which formulation is optimal for DMXB-A, without the confound of differences in pharmacodynamic properties.

Heretofore, DMXB-A has been tested only in non-smoking patients, because of the potential for interference from the desensitization produced by nicotine and the possibility of increased adverse effects resulting from the simultaneous presence of both nAChR agonists. Industry trials have included smokers, but they have not assessed smoking itself to see whether the introduction of an agonist also changed smoking behavior, which indirectly might contribute to the cognitive effects. We therefore recruited half the subjects to be smokers to assess the effect of the slower release formulation on both their cognition and their smoking, measured as their nicotine and cotinine plasma levels at the conclusion of the 4-week treatment.

\section{MATERIALS AND METHODS}

\section{Subjects}

One hundred thirty-eight patients were screened. The most common exclusion was marijuana abuse. Eighty patients age 18-60 years with schizophrenia by DSM-IV criteria and SCID interview were enrolled, 37 non-smokers and 43 smokers (Table 1; Supplementary Figure S1). All subjects gave informed consent for the study.

Patients were currently being treated with a wide range of antipsychotic drugs in usual therapeutic doses, except for clozapine; treatment with clozapine was an exclusion criterion because it often alters inhibition of the P50evoked potential, a biomarker for attention (Nagamoto et al, 1996). Two participants were not taking antipsychotic medication. All had been on the same antipsychotic regimen for at least 3 months without change in their clinical status. Their drug regimens were maintained during the trial. No patient required a change for clinical reasons. Exclusion criteria were history of severe head injury with neurocognitive disturbance, and positive urinary toxicology screen for drugs of abuse including marijuana. Female patients had an assured method of birth control and a negative pregnancy screen.

Patients were sub-grouped into smokers who smoked at least 10 cigarettes per day and non-smokers, who had not smoked for at least 1 month. Non-smoking status was verified by expired CO level.
Table I Demographic Data

\begin{tabular}{lcccc}
\hline & $\begin{array}{c}\text { DMXB-A mean } \\
\text { or } \mathbf{N}\end{array}$ & SD & $\begin{array}{c}\text { Placebo mean } \\
\text { or } \mathbf{N}\end{array}$ & SD \\
\hline Age & 46.5 & 12.4 & 43.2 & 13.2 \\
Male & 32 & & 28 & \\
Education & 12.4 & 2.4 & 13.6 & 2.1 \\
Caucasian $(N)$ & 31 & & 27 & \\
African-American $(N)$ & 6 & & 10 & \\
Hispanic $(N)$ & 1 & & 3 & \\
Other $(N)$ & 2 & & 0 & \\
Schizoaffective $(N)$ & 7 & & 12 & \\
Smokers $(N)$ & 21 & & 22 & \\
Packs per day & 1.1 & 0.6 & 1.1 & \\
Total subjects $(N)$ & 40 & & 40 & \\
\hline
\end{tabular}

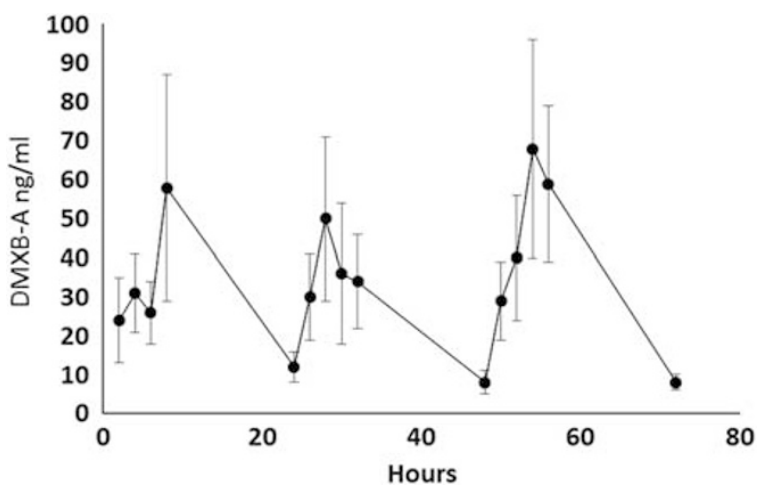

Figure I Mean and SD of 3-(2,4-dimethoxybenzylidene)-anabaseine (DMXB-A) plasma level during administration of $150 \mathrm{mg}$ of the hypromellose formulation (DMXBA-ER) four times daily. There were $N=10$ normal, non-smoking subjects who were not in the clinical trial.

\section{Drug Formulation}

3-(2,4-dimethoxybenzylidene)-anabaseine (DMXB-A) was synthesized as previously described (Olincy et al, 2006). For the extended-release preparation (DMXBA-ER), DMXBA $150 \mathrm{mg}$ was mixed with hypromellose to fill a standard \#1 capsule. Hypromellose is also used to formulate quetiapine for extended release. Automated filling and sealing techniques were employed. A preliminary study in 10 healthy non-smoking subjects who were not subjects in the subsequent clinical trial showed the time curve for change in the plasma concentration of DMXB-A. The mean level slowly increased between 1 and $4 \mathrm{~h}$ after administration (Figure 1). After administration four times daily, as was used in the current trial, no subject had levels before the next morning's dose $>5 \mathrm{ng} / \mathrm{ml}$. Thus, most DMXB-A is cleared from the plasma overnight.

\section{Conduct of the Trial}

The trial was approved by the Colorado Multi-Institutional Review Board. The trial was a parallel comparison between placebo and $150 \mathrm{mg}$ DMXB-A-ER in equal numbers of subjects. All treatments and assessments were performed 
under double-blind conditions at the University of Colorado from August 1, 2011 through June 1, 2015. Side effects and vital signs were monitored weekly, along with pill counts. All subjects received open-label placebo for 1 week. Subjects who could not maintain $70 \%$ compliance during that week were required to repeat the week to obtain compliance. Medication boxes were monitored weekly during the trial and compliance was encouraged. The MATRICS Consensus Cognitive Battery (MATRICS CCB), the primary outcome, and the secondary outcomes, BPRS, SANS (Sum of Global Ratings in Associality/Anhedonia, Affective Flattening, Avolition/Apathy, Alogia), and P50 auditory-evoked potential inhibition, were assessed at 4 weeks. Previously published methodology was used for these assessments (Olincy et al, 2006; Freedman et al, 2008; Nuechterlein et al, 2008). The planned enrollment was completed.

\section{Plasma Drug Assays}

Plasma specimens for drug level assays were obtained at $2 \mathrm{~h}$ after the first or second morning dose, after neurocognitive testing. The specimens were analyzed by high-performance liquid chromatography as previously described (Mahnir et al, 1998). DMXB-A's 4-hydroxy metabolite was generally below the level of reliable quantification.

\section{Randomization and Statistical Analysis}

Separate randomization schemes were used for smokers and non-smokers, to ensure equal numbers of drug and placebo treatments within each subgroup. The planned trial enrollment of 20 subject per group (DMXBA-ER or placebo, smokers or non-smoker) was determined by a power analysis (alpha 2 tailed $0.05,1$-beta $=0.8$ based on the previous determination of DMXB-A's effect size $\left(d^{\prime}=0.97\right)$ on the mean of MATRCS-CCB Neurocognitive domains (Freedman et al, 2008). Randomization was performed by the statistician using SAS and given to the pharmacists, who were the only non-blinded study personnel. The allocation sequence was not available to other personnel. The primary outcome measure was the MATRICS CCB Neurocognitive Composite, which encompasses all domains except Social Cognition (MATRICS Assessment Inc., 2015). An analysis of variance with sex, age, and baseline performance as covariates was performed for MATRICS CCB Neurocognitive Composite T-scores for assessment after 4 weeks of treatment. Protected univariate analyses were performed for each subgroup of smokers and non-smokers. All other exploratory analyses were performed with Student's $t$-tests. Reported significance levels are two-tailed.

\section{Comparison with an Immediate-Release Formulation}

An exploratory post hoc analysis to compare the effects of extended exposure (DMXBA-ER) with the previously studied immediate-release formulation (DMXBA-IR, Freedman et al, 2008) was conducted. The MATRICS CCB Neurocognitive Composite Index was not available at the time of the publication of that study. That Composite Index has now been computed. The earlier study had a three-arm crossover design with all subjects receiving DMXBA-IR $75 \mathrm{mg}, 150 \mathrm{mg}$, and placebo. Data were previously analyzed from the first arm of the crossover compared with the baseline because of significant effects of repeated MATRICS CCB testing over time. For the post hoc analysis, the 16 patients who received DMXBA-IR in the first arm at either dose were combined. The mean DMXB-A plasma levels were skewed in both the present extended-release study and in the earlier immediaterelease study, with one subject in each formulation group having plasma levels over three SDs above the mean. In the DMXBA-IR study, the subject had plasma levels three SDs above the mean for both doses. We hypothesize that these individuals failed to $O$-demethylate one or both of the DMXB-A methoxy groups, conversions that promote both agonist effects and excretion (Azuma et al, 1999; Kem et al, 2004). Therefore, the comparative analyses were conducted omitting these two individuals. None of the patients studied with the immediate-release formulation was smokers. For the extended-release formulation, only the non-smoking group was analyzed for the comparison.

\section{RESULTS}

Eighty patients with schizophrenia were studied for 4 weeks: 40 received extended-release DMXB-A $150 \mathrm{mg}$ qid for 4 weeks and 40 received placebo. Sub-grouped by smoking status, 18 non-smokers received placebo and 19 received extended-release DMXB-A-ER; 21 smokers received placebo and 22 received DMXB-A-ER. One patient left the DMXBAER group because he had a transient ischemic episode. During evaluation in the Emergency Department, he revealed a history of several such episodes with similar clinical appearance, which he had not previously disclosed. His attack was not ascribed to his experimental treatment. A second patient withdrew after randomization and did not return for further evaluation. Reports of other adverse effects showed no significant differences between groups. The most common side effects in non-smokers who received DMXBA-ER were gastrointestinal, including nausea, diarrhea, flatulence, and constipation. None was severe. These effects were not observed in smokers who received DMXB-A-ER. There was also a similar distribution of laboratory values outside the normal range for both groups. None was considered to be medically significant (Supplementary Tables 1). Neither DMXB-A-ER nor placebo groups displayed significant changes with weight, $0.8 \pm 6.0 \mathrm{lbs}$ for DMXB-A-ER and $-0.3 \pm 6.0 \mathrm{lbs}$ for placebo.

\section{Neurocognitive and Symptomatic Outcome}

The primary outcome was the MATRICS CCB Neurocognitive Composite $\mathrm{T}$ score. An analysis of variance with age, sex, and pre-treatment baseline as covariates showed no significant relationship with any of these variables. The mean change from baseline for the non-smoker and smoker groups combined was $2.10 \pm 8.24$ for placebo and $0.64 \pm 5.82$ for DMXB-A-ER. The practice effect in the placebo group was non-significant $(t=0.73$, df $39, p=0.47)$. Subgroup analysis showed no effect in the non-smoking patients (placebo $2.78 \pm 5.98$ and DMXB-A-ER $1.06 \pm 6.26$ ) and no effect in the smoking patients (placebo $1.52 \pm 9.90$ and DMXB-A-ER $0.29 \pm 5.43$ ). No significant effects of treatment were observed on any MATRICS CCB individual domain. 
Clinical ratings did not change significantly after DMXBA-ER treatment: mean change in BPRS DXMBA-ER $-3.11 \pm 8.07$, placebo $-2.17 \pm 4.63$; mean change in SANS DMXBA-ER $0.22 \pm 1.56$, placebo $0.71 \pm 1.81$.

Inhibition of the P50 auditory-evoked potential was measured to assess whether DMXB-A-ER had its intended neurobiological effect. In the previous DMXB-A-IR trial, this had shown significant increase in inhibition with DMXBAIR compared with placebo (Olincy et al, 2006). In the current trial, neither the smokers nor the non-smokers showed significant effects of DMXBA-ER on P50 inhibition, compared with placebo: DMXBA-ER P50 ratio $0.50 \pm 0.56$ compared with the placebo $0.39 \pm 0.32$.

There were no statistically significant differences in nicotine and cotinine levels in smokers with treatment. Nicotine levels were $14 \pm 13 \mathrm{ng} / \mathrm{ml}$ during placebo treatment and $19.8 \pm 16.1 \mathrm{ng} / \mathrm{ml}$ during DMXBA-ER. Cotinine levels were $122 \pm 39 \mathrm{ng} / \mathrm{ml}$ during placebo treatment and $113.7 \pm 39.3 \mathrm{ng} / \mathrm{ml}$ during DMXBA-ER.

Plasma levels of DMXB-A were an order of magnitude lower in smokers than in non-smokers. The levels were drawn at the time of cognitive testing, about $2.5 \mathrm{~h}$ after the morning dose. For non-smokers, the mean level was $46.7 \pm 62.3 \mathrm{ng} / \mathrm{ml}$ and for smokers it was $4.2 \pm 3.7 \mathrm{ng} / \mathrm{ml}$.

\section{Exploratory Comparison with the Immediate-Release Formulation}

There was no significant difference in DMXB-A plasma levels between DMXB-ER $(56.2 \pm 96 \mathrm{ng} / \mathrm{ml})$ and DMXBA-IR $(70.1 \pm 63.5 \mathrm{ng} / \mathrm{ml} ; t=0.47$, df $28, p=0.64)$. The post hoc comparison of the effects on the MATRICS-CCB obtained with the DMXBA-ER and DMXBA-IR found that the Neurocognitive Index T-scores were significantly elevated compared with the baseline for DMXBA-IR $(4.20 \pm 3.17$ T-score units, $t=5.31$, df 15, $p<0.0001)$. By comparison, the effect of DMXBA-ER was not significantly greater than baseline values $(1.06 \pm 6.26$ T-score units). The most significant effect of DMXBA-IR had previously been found to be on the Attention-Vigilance Domain (Freedman et al, 2008). The effect of DMXBA-IR on this domain (7.80 \pm 8.85 T-score units) was significantly greater than the effect of DMXBA-ER $(-1.83 \pm 6.46 \mathrm{~T}$ score units, $t=3.61$, df 31 , $p=0.0011$; Figure 2). The placebo change in the Neurocognitive Index T-score with placebo was similar in the two trials $(2.78 \pm 5.98 \mathrm{~T}$ score units in the DMXBA-ER trial and $2.11 \pm 6.27$ in the DMXBA-IR trial; Table 2).

\section{DISCUSSION}

This is the first clinical trial that permits direct comparison of the effects of immediate-release and extended-release oral formulations of the same alpha7-nAChR agonist in schizophrenia. The cognitive effects observed with a brief-acting formulation were not seen with a longer acting formulation, despite nearly comparable plasma levels during testing. These results are consistent with the known physiological properties of alpha7-nAChRs, which display more extensive desensitization during prolonged exposure to agonists (Peng et al, 1994). They suggest that long duration-acting drugs are

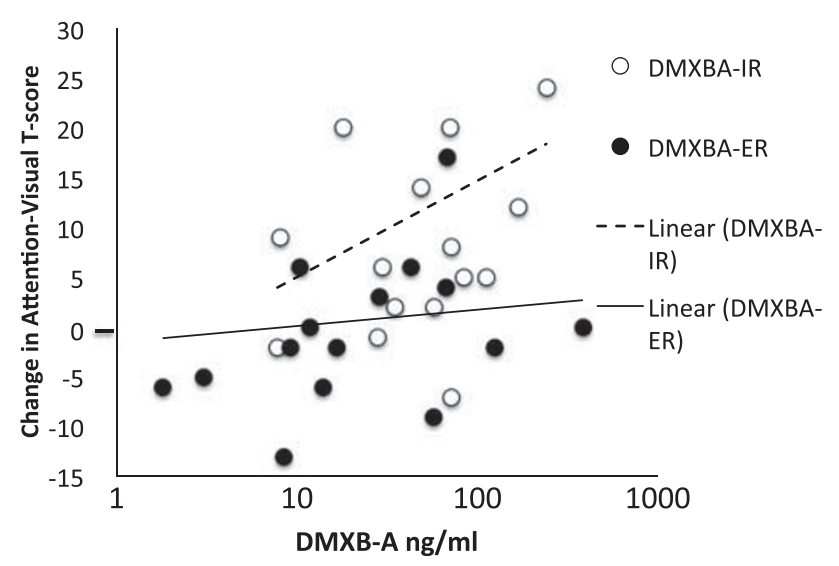

Figure 2 Comparison of treatment for I month with DMXBA-IR and DMXBA-ER formulation on the Attention-Vigilance Domain of the MATRICS-CCB in non-smoking schizophrenia patients. The effect of DMXBA-IR was significantly greater than the effect of DMXBA-ER (see text). The trendlines are illustrative only; neither is significant.

not likely to be effective for ameliorating neurocognitive deficits in schizophrenia.

The current trial demonstrates that even with a partial agonist, prolonged exposure to an alpha7-nAChR agonist is not an effective treatment strategy. A weak partial agonist like DMXB-A is an appropriate molecule for the investigation of pharmacokinetic limitations of alpha7-nAChR stimulation, because it is less likely to produce chronic desensitization than a stronger partial or full agonist that increases the probability that the activated receptors are converted into a stable desensitized state, rather than back into a resting state that can reactivated by agonist (Papke et al, 2009). Desensitization is intimately tied to receptor activation, so that full agonists have robust immediate effects, but they then produce more desensitization with loss of effect (Quick and Lester, 2002). Inverted U-shaped dose-response curves, indicative of desensitization-induced tachyphylaxis at higher agonist doses, have been observed in investigations of the effects of alpha7 agonists on cognitive and related prefrontal cortical functions in primates (Yang et al, 2013). Loss of pro-cognitive effect with chronic exposure to nicotine, a full agonist, is also well known. Although smoking produces short-duration bursts of very high nicotine levels, smokers' plasma levels of nicotine are chronically elevated. In earlier clinical tests with nicotine, we were unable to demonstrate any cognitive effect of acute nicotine, whether smoked or administered as nicotine gum, in schizophrenia patients who were chronic smokers, whereas acutely administered nicotine produced cognitive effects in non-smoking patients (Harris et al, 2004). Alpha7 receptors show some compensatory upregulation during chronic exposure to nicotinic agonists, but the effect is relatively small in comparison with the upregulation of alpha4-beta2 receptors (Lee et al, 2001).

In the current trial, the particularly low levels of DMXB-A in smokers were an additional confounding factor. Hepatic metabolism has been shown to be the limiting factor in the bioavailability of DMXB-A (Mahnir et al, 1998; Azuma et al, 1999). The metabolic profile for DMXB-A obtained in vitro shows predominantly $O$-demethylation at the 4-methoxy 
Table 2 Comparison of Results From Trials of DMXBA-ER and DMXBA-IR in Non-Smokers and DMXBA-ER in Smokers, Means (SD)

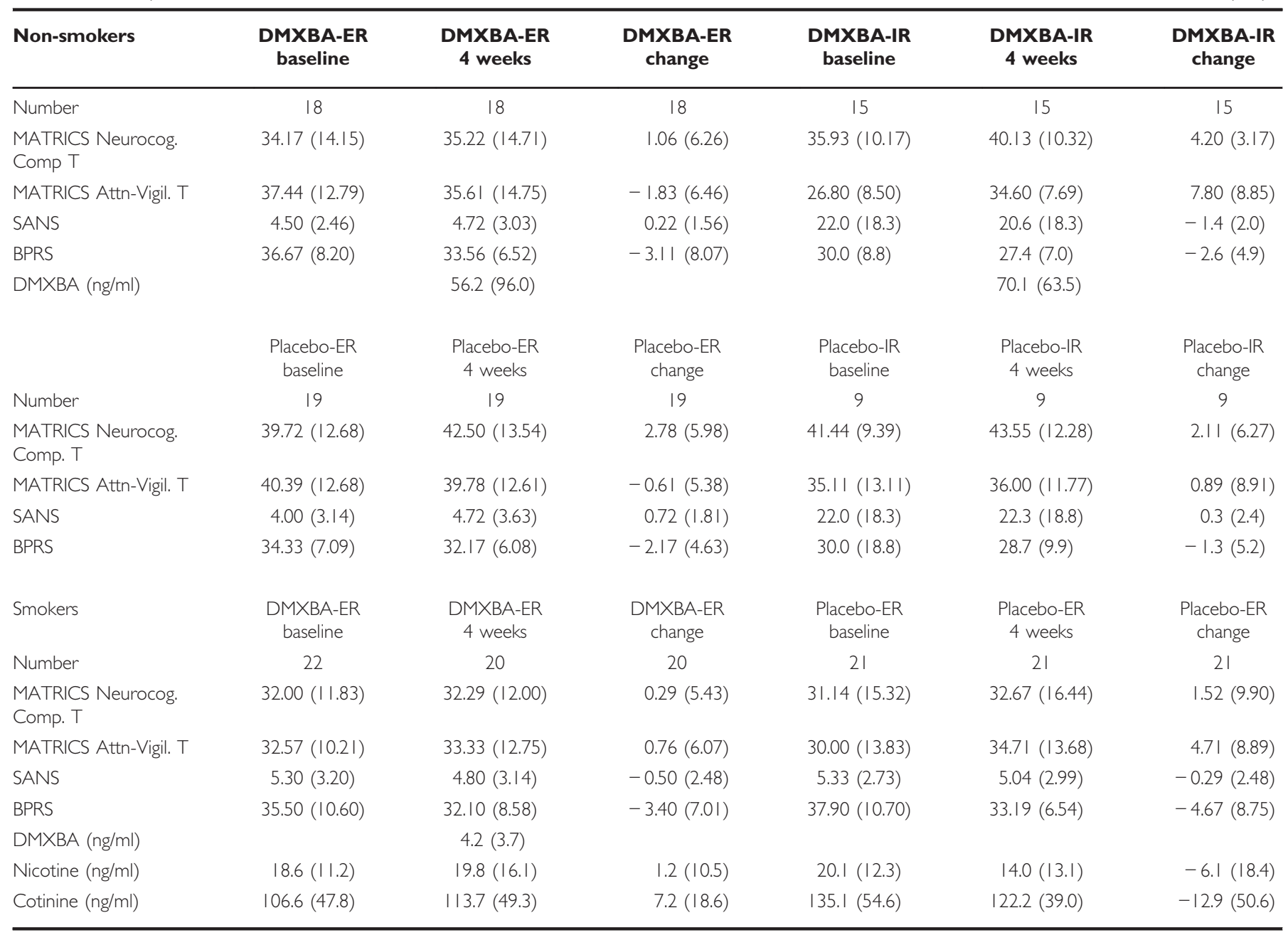

position of the benzylidene ring. This reaction is catalyzed by three different families of cytochrome P450 enzymes, including CYP1A1 and CYP1A2 (Azuma et al, 1999; Kem et al, 2004). Hepatic levels of these particular cytochrome P450 enzymes are increased by chronic cigarette smoking (Zevin and Benowitz, 1999), which may be the major explanation for the drastically reduced plasma levels of DMXB-A in the smoking group in our study.

The major finding relevant for future drug development comes from the exploratory post hoc comparisons with the results of the previously published study of the immediaterelease formulation, using the newly available $\mathrm{MCCB}$ Neurocognitive Composite. The re-formulation of DMXBA to obtain more extended levels led to the loss of such effects observed previously with the short immediate-release formulation. Plasma drug levels were determined immediately after MATRICS-CCB testing for both formulations, but the higher transient peak levels achieved by the faster release from the DMXBA-IR formulation and the subsequent rapid elimination are the likely reason for its greater effect. A limitation is that the comparison of DMXBA-ER and DMXBA-IR was not conducted in the same trial. However, the placebo response rates in the two trials, conducted at the same site, were quite similar. The comparison of the two formulations cannot fully distinguish between the alternative explanations that a high initial peak of activation is required, best achieved with immediate release of the agonist, compared with the possibility that the subsequent prolonged exposure from the extended-release formulation results in tachyphylaxis of the response.

Pharmaceutical companies that have developed agonist therapies for alpha7-nicotinic receptor in schizophrenia have selected drug candidates with long half-lives for once daily administration (Lieberman et al, 2013; Keefe et al, 2015; Haig et al, 2016a, 2016b). These drug candidates have produced promising results in initial, phase 2 trials, which have not been corroborated in the subsequent larger studies. DMXBA-IR produced effects on the MATRICS-CCB Domains consistent with a meaningful clinical effect (Freedman et al, 2008). The increase of $4.20 \pm 3.17$ T-score units with DMXBA-IR is equivalent to the $4.8 \pm 1.8$ increase associated with employment in patients with schizophrenia, compared to patients who are not employed (August et al, 2012). Phasic stimulation may be particularly effective because DMXB-A activates a nitric-oxide-mediated second messenger response 
in neurons that appears to extend its effect after briefreceptor stimulation (Adams et al, 2000).

A model for this interpretation is the cholinergic crisis or overstimulation produced by the extended presence of acetylcholine at neuromuscular nicotinic receptors in myasthenia gravis. Both myasthenia gravis and schizophrenia have in common a diminished expression of the relative nicotinic receptor, although the depletion is more severe in myasthenia (Freedman et al, 1995; Court et al, 1999; Guillozet-Bongaarts et al, 2014). The findings suggest that either judicious use of agonists, employing brief half-life drugs intermittently, or allosteric modulators to enhance the effects of phasically released acetylcholine may be required to achieve therapeutically significant cognitive effects through alpha7-nAChR receptor stimulation in schizophrenia (Gee et al, 2017).

\section{FUNDING AND DISCLOSURE}

This trial was funded by NIH Grant P50MH086383 and the Department of Veterans Affairs Medical Research Service. WRK is a co-inventor on a University of Florida patent for DMXB-A. The other authors have no financial conflicts of interest. Clinical Trial Registration NCT01400477. Conducted under FDA IND exemption 105,448. Colorado Multi-Institutional Review Board approval number 11-0459.

\section{REFERENCES}

Adams CE, Stevens KE, Kem WR, Freedman R (2000). Inhibition of nitric oxide synthase prevents alpha 7 nicotinic receptor-mediated restoration of inhibitory auditory gating in rat hippocampus. Brain Res 877: 235-244.

August SM, Kiwanuka JN, McMahon RP, Gold JM (2012). The MATRICS Consensus Cognitive Battery (MCCB): clinical and cognitive correlates. Schizophr Res 134: 76-82.

Azuma R, Komuro M, Korsch BH, Andre JC, Onnagawa O, Black SR et al (1999). Metabolism and disposition of GTS-21, a novel drug for Alzheimer's disease. Xenobiotica 29: 747-762.

Briggs CA, McKenna DG, Papke RL, Kem WR, Soti F, López-Hernández GY et al (1998). Activation and inhibition of the human alpha7 nicotinic acetylcholine receptor by agonists. Neuropharmacology 37: 1095-1102.

Court J, Spurden D, Lloyd S, McKeith I, Ballard C, Cairns N et al (1999). Neuronal nicotinic receptors in dementia with Lewy bodies and schizophrenia: alpha-bungarotoxin and nicotine binding in the thalamus. J Neurochem 73: 1590-1597.

Freedman R, Hall M, Adler LE, Leonard S (1995). Evidence in postmortem brain tissue for decreased numbers of hippocampal nicotinic receptors in schizophrenia. Biol Psychiatry 38: 22-33.

Freedman R, Olincy A, Buchanan RW, Harris JG, Gold JM, Johnson L et al (2008). Initial phase 2 trial of a nicotinic agonist in schizophrenia. Am J Psychiatry 165: 1040-1047.

Gee KW, Olincy A, Kanner R, Johnson L, Hogenkamp D, Harris J et al (2017). First in human trial of a type I positive allosteric modulator of alpha7-nicotinic acetylcholine receptors: pharmacokinetics, safety, and evidence for neurocognitive effect of AVL-3288. J Psychopharmacol 31: 434-441.

Guillozet-Bongaarts AL, Hyde TM, Dalley RA, Hawrylycz MJ, Henry A, Hof PR et al (2014). Altered gene expression in the dorsolateral prefrontal cortex of individuals with schizophrenia. Mol Psychiatry 19: 478-485.
Haig G, Bain E, Robieson W, Baker J, Othman A (2016a). A randomized, double-blind trial to assess the efficacy and safety of ABT-126, a selective $\alpha 7$ nicotinic acetylcholine receptor agonist in the treatment of cognitive Impairment in subjects with schizophrenia. Am J Psychiatry 173: 827-835.

Haig G, Wang D, Othman AA, Zhao J (2016b). The $\alpha 7$ nicotinic agonist ABT-126 in the treatment of cognitive impairment associated with schizophrenia in nonsmokers: results from a randomized controlled phase $2 \mathrm{~b}$ study. Neuropsychopharmacology 41: 2893-2902.

Harris JG, Kongs S, Allensworth D, Martin L, Tregellas J, Sullivan B et al (2004). Effects of nicotine on cognitive deficits in schizophrenia. Neuropsychopharmacology 29: $1378-1385$.

Keefe RSE, Meltzer HA, Dgetluck N, Gawryl M, Koenig G, Moebius $\mathrm{HJ}$ et al (2015). Randomized, double-blind, placebo-controlled study of encenicline, an $\alpha 7$ nicotinic acetylcholine receptor agonist, as a treatment for cognitive impairment in schizophrenia. Neuropsychopharmacology 40: 3053-3060.

Kem WR, Mahnir VM, Prokai L, Papke RL, Cao X, LeFrancois S et al (2004). Hydroxy metabolites of the Alzheimer's drug candidate 3-[(2,4-dimetoxy) benzylidene]-anabaseine dihydrochloride (GTS-21): their molecular properties, interactions with brain nicotinic receptors, and brain penetration. Mol Pharmacol 65: 56-67.

Kitagawa H, Takenouchi T, Azuma R, Wesnes KA, Kramer WG, Clody DE et al (2003). Safety, pharmacokinetics, and effects on cognitive function of multiple doses of GTS-21 in healthy, male volunteers. Neuropsychopharmacology 28: 542-551.

Lee MJ, Breese CR, Strook ML, Leonard S (2001). The effect of nicotine and haloperidol co-treatment on nicotinic receptor levels in the rat brain. Mol Brain Res 86: 115-124.

Lieberman JA, Dunbar G, Segreti AC, Girgis RR, Seoane F, Beaver JS et al (2013). A randomized exploratory trial of an alpha-7 nicotinic receptor agonist (TC-5619) for cognitive enhancement in schizophrenia. Neuropsychopharmacology 38: 968-975.

Lopez-Hernandez GY, Thinschmidt JS, Morain P, Trocme-Thibierge C, Kem WR, Soti F et al (2009). Positive modulation of alpha7 nAChR responses in rat hippocampal interneurons to full agonists and the alpha7-selective partial agonists, 4OH-GTS-21 and S S24795. Neuropharmacology 56: 821-830.

Mahnir VM, Lin B, Prokai-Tatrai K, Kem WR (1998). Pharmacokinetics and urinary excretion of DMXBA (GTS-21), a compound enhancing cognition. Biopharm Drug Dispos 19: 147-151.

MATRICS Assessment Inc (2015). A change in the MCCB scoring system. Available at http://www.matricsinc.org/?page_ id $=25$. (Last downloaded September 7, 2017.)

Nagamoto HT, Adler LE, Hea RA, Griffith JM, McRae KA, Freedman R (1996). Gating of auditory P50 in schizophrenics: unique effects of clozapine. Biol Psychiatry 40: 181-188.

Nuechterlein KH, Green MF, Kern RS, Baade LE, Barch DM, Cohen JD et al (2008). The MATRICS Consensus Cognitive Battery, part 1: test selection, reliability, and validity. Am J Psychiatry 165: 203-213.

Olincy A, Harris JG, Johnson LL, Pender V, Kongs S, Allensworth D et al (2006). Proof-of-concept trial of an $\alpha 7$ nicotinic agonist in schizophrenia. Arch Gen Psychiatry 63: 630-638.

Papke RL, Kem WR, Soti F, López-Hernández GY, Horenstein NA (2009). Activation and desensitization of nicotinic alpha7-type acetylcholine receptors by benzylidene anabaseines and nicotine. $J$ Pharmacol Exp Ther 329: 791-807.

Peng X, Katz M, Gerzanich V, Anand R, Lindstrom J (1994). Human alpha 7 acetylcholine receptor: cloning of the alpha 7 subunit from the SH-SY5Y cell line and determination of pharmacological properties of native receptors and functional alpha 7 homomers expressed in Xenopus oocytes. Mol Pharmacol 45: 546-554. 


\section{Alpha7-nicotinic agonist effects in schizophrenia}

WR Kem et al

Quick MW, Lester RA (2002). Desensitization of neuronal nicotinic receptors. J Neurobiol 53: 457-478.

Umbricht D, Keefe RS, Murray S, Lowe DA, Porter R, Garibaldi G et al (2014). A randomized, placebo-controlled study investigating the nicotinic alpha7 agonist, RG3487, for cognitive deficits in schizophrenia. Neuropsychopharmacology 39: 1568-1577.

Walling D, Marder SR, Kane J, Fleischhacker WW, Keefe RS, Hosford DA et al (2016). Phase 2 trial of an alpha-7 nicotinic receptor agonist (TC-5619) in negative and cognitive symptoms of schizophrenia. Schizophr Bull 42: 335-343.

Yang Y, Paspalas CD, Jin LE, Picciotto MR, Arnsten AFT, Wang M (2013). Nicotinic alpha7 receptors enhance NMDA cognitive circuits in dorsolateral prefrontal cortex. Proc Natl Acad Sci USA 110: $12078-12083$.

Zevin S, Benowitz NL (1999). Drug interactions with tobacco smoking. Clin Pharmacokinet 36: 425-436.

Supplementary Information accompanies the paper on the Neuropsychopharmacology website (http://www.nature.com/npp) 